\title{
Exploring Theranostic Potentials of Radioiodinated Hypericin in Rodent Necrosis Models
}

\author{
Junjie Li ${ }^{1,2}$, Marlein Miranda Cona ${ }^{1,2}$, Feng Chen ${ }^{1,2}$, Yuanbo Feng ${ }^{1,2}$, Lin Zhou ${ }^{3}$, Jie $\mathbf{Y u}^{1}$, Johan Nuyts ${ }^{3}$, Peter \\ de Witte ${ }^{4}$, Jian Zhang ${ }^{5}$, Uwe Himmelreich², Alfons Verbruggen ${ }^{4}$, Yicheng Ni1,2,5凶 \\ 1. Theragnostic Laboratory, Department of Imaging \& Pathology, Biomedical Sciences Group, KU Leuven, Herestraat 49, Leuven, Belgium. \\ 2. Molecular Small Animal Imaging Centre/MoSAIC, Biomedical Sciences Group, KU Leuven, Herestraat 49, Leuven, Belgium. \\ 3. Nuclear Medicine \& Medical Imaging, Department of Imaging \& Pathology, Faculty of Medicine, Biomedical Sciences Group, KU Leu- \\ ven, Herestraat 49, Leuven, Belgium. \\ 4. Faculty of Pharmaceutical Sciences, Biomedical Sciences Group, KU Leuven, Herestraat 49, Leuven, Belgium. \\ 5. Laboratory of Translational Medicine, Jiangsu Academy of Traditional Chinese Medicine, Nanjing 210028, Jiangsu Province, China
}

$\triangle$ Corresponding author: Prof. Yicheng Ni. Theragnostic Laboratory, KU Leuven, Herestraat 49, BE-3000 Leuven, Belgium. Tel: +32-16-33 01 65, Fax: +32-16-34 37 65. E-mail: Yicheng.ni@med.kuleuven.be

() Ivyspring International Publisher. This is an open-access article distributed under the terms of the Creative Commons License (http://creativecommons.org/ licenses/by-nc-nd/3.0/). Reproduction is permitted for personal, noncommercial use, provided that the article is in whole, unmodified, and properly cited.

Received: 2012.07.25; Accepted: 2012.08.17; Published: 2012.10.18

\begin{abstract}
Objectives: The present animal experiments were conducted to evaluate radioiodinated Hypericin (Hyp) for its regional distribution as well as theranostic potentials.

Materials and Methods: Rat models of reperfused liver infarction (RLI) and hepatic rhabdomyosarcoma $(\mathrm{RI})$ were surgically induced. $\mathrm{RI}$ models received Combretastatin A4 phosphate (CA4P) intravenously at $10 \mathrm{mg} / \mathrm{kg} 24 \mathrm{~h}$ prior to radioiodinated Hyp. Three groups of 6 rats each containing $3 \mathrm{RLI}$ and $3 \mathrm{RI}$ models received iv injections of ${ }^{123} \mathrm{I}-\mathrm{Hyp}$ at 37, 74, and I 85 $\mathrm{MBq} / \mathrm{kg}$ respectively and followed by $0.1 \mathrm{ml}$ of I\% Evans blue solution were sacrificed at 4, 24 and 48 hour post injection immediately after in vivo examination of MRI and planar gamma scintigraphy. Besides, two groups of $6 \mathrm{RI}$ models that received either $300 \mathrm{MBq} / \mathrm{kg}$ of ${ }^{131} \mathrm{I} \mathrm{I}-\mathrm{Hyp}$ or vehicle intravenously were examined using MRI to compare tumor growth for 12 days. Autoradiography, gamma counting, and histopathology were performed for postmortem verifications and quantification.

Results: Necrosis as seen in vivo on contrast-enhanced MRI corresponded well with the hot spots on planar scintigraphy. Autoradiography and gamma counting revealed intense accumulation of ${ }^{123} \mathrm{I}-\mathrm{Hyp}$ in necrotic liver $(3.94 \pm 1.60,5.38 \pm \mathrm{I} .04$, and $6.03 \pm 2.09 \% \mathrm{ID} / \mathrm{g} \pm \mathrm{SD})$ and necrotic tumor $(4.27 \pm 0.76,5.57 \pm 0.76$, and $5.68 \pm \mathrm{I} .33 \% \mathrm{ID} / \mathrm{g} \pm \mathrm{SD})$ relative to normal liver $(\mathrm{I} .76 \pm 0.54,0.4 \mathrm{I} \pm 0.18$, and $0.16 \pm 0.07 \% \mathrm{ID} / \mathrm{g} \pm \mathrm{SD})$, with a high necrosis-to-liver ratio of $2.3, I 4.0$, and 37.0 at 4,24 and $48 \mathrm{~h}$ respectively. Tumor volumes in RI models that received ${ }^{131} \mathrm{I}$-Hyp and vehicle changed from $0.45 \pm 0.09$, and $0.47 \pm 0.12 \mathrm{~cm}^{3}(p>0.05)$ on day 0 tol. 32 \pm 0.76 and $3.63 \pm 0.72 \mathrm{~cm}^{3}(\mathrm{p}<0.00 \mathrm{I})$ on day 12 , with the corresponding necrosis ratios from $73 \pm 12 \%$, and $76 \pm 17 \%$ to $47 \pm 18 \%$ and $17 \pm 13 \%(p<0.01)$, and with the tumor DT of 7.3 \pm 1.0 and $4.2 \pm 0.7$ days, respectively.

Conclusions: Radioiodinated Hyp as a necrosis avid tracer appears promising for non-invasive imaging diagnosis of necrosis-related pathologies. Its prominent targetability to necrosis allows targeted radiotherapy for malignancies on top of a prior necrosis-inducing treatment.
\end{abstract}

Key words: Radioiodinated Hypericin, Theranostic, Necrosis avidity, Malignancies. 


\section{Introduction}

Necrosis that refers to the death of cells or tissues from severe injury or disease is a principal feature of numerous pathologies or disorders [1]. Imaging necrotic tissue may potentially improve the diagnosis of a wide variety of diseases such as pancreatitis, malignancies, stroke, myocardial infarction and drug induced cell or organ toxicity etc, caused by inflammation, hypoxia, infection, ischemia, atherosclerosis, hypoglycaemia, toxins, sepsis, and dysfunction $[2,3]$. Necrosis avid contrast agents (NACAs) have been explored over the past decade for determining disease severity, estimating prognosis, monitoring therapeutic responses, and formulating therapeutic strategies $[4,5]$. Therapeutic radio-conjugations of NACAs are expected to be anchored by tumoral necrosis for destroying neighboring viable tumor cells [6,7]. Challenge exists in finding NACAs with extraordinarily high avidity and specificity for necrosis [4].

Hypericin (Hyp), or chemically 4,5,7,4',5',7'-Hexahydroxy-2,2'-dimethylnaphthodiant hrone, can be either extracted from the plant Hypericum perforatum, or semi-synthesized from emodin anthraquinone [8-11]. Hyp was recently recognized as one of the nonporphyrin NACAs with exceptional necrosis affinity [8]. Radio-derivates of Hyp such as ${ }^{123} \mathrm{I}$-iodohypericin ( $\left.{ }^{123} \mathrm{I}-\mathrm{Hyp}\right), \quad{ }^{64} \mathrm{Cu}$-Bis-DOTAHypericin, were investigated as NACAs for diagnosis of various diseases [9-13]. Preclinical studies have reported target-to-nontarget ratios about 10-80 with radioiodinated Hyp, which are much higher than the ever-discovered specific agents $[4,8,11]$. As mentioned above, the exploration of necrosis avid Hyp constitutes a potential strategy not only for detecting various diseases, but also for developing radiotherapeutic agents to target malignancies. More recently, ${ }^{131}$ I-iodohypericin (131I-Hyp) was explored as a necrosis targeting theragnostic agent for management of malignancies [14-17]. A novel anticancer approach called small-molecular sequential dual-targeting theragnostic strategy (SMSDTTS), which combines the sequential use of a vascular disrupting agent (VDA) Combretastatin A4 phosphate (CA4P) and a necrosis avid tracer 131I-Hyp, was introduced in a proof-of-concept research with encouraging results from rodent tumor models [15-17].

Although promising, previous studies with radioiodinated Hyp have left rooms for improvement, e.g., the micro-geographical distributions inside necrotic tissues are yet to explore for better comprehension and application of its theranostic capacity. Some questions need to be answered. For examples, which part of the necrosis is most likely for Hyp to enter?
How long can Hyp stick to necrosis? What are the regional distribution patterns of Hyp along with tumor growth? Can residual tumor cells really be eradicated by only a single dose treatment using ${ }^{131}$ I-Hyp? We therefore performed the present experiments to gain more insight into these aspects. The potentials of both 123I-Hyp and 131I-Hyp for diagnosing bulky necrotic tissues, detecting and targeting solid tumors were quantitatively evaluated and discussed in animal models of pathologies.

\section{Materials and methods}

\section{Animal models of pathologies}

All animal studies were approved by the institutional animal care and research advisory committees. Thirty WAG/Rij rats weighting around $300 \mathrm{~g}$ were recruited for the experiments.

Rat models of reperfused liver infarction (RLI): Nine rats were anesthetized with intra-peritoneal injection of pentobarbital (Nembutal; Sanofi Sante Animale, Brussels, Belgium) at a dose of $40 \mathrm{mg} / \mathrm{kg}$. Under laparotomy, the hilum of the right liver lobe was clamped for $3 \mathrm{~h}$ to induce liver infarction. Reperfusion was assured by declamping hepatic inflow and by giving massage to the liver lobe, the abdominal cavity was closed with two layered sutures, and the animals were allowed to recover for at least $8 \mathrm{~h} \mathrm{[18].} \mathrm{The} \mathrm{co-}$ existing infarcted and normal liver lobes were used as their own control for intra-individual comparison.

Rat models of hepatic allograft rhabdomyosarcoma (R1): R1 tissues from an adult rat were used as donor. Twenty-one rats underwent midline laparotomy after being anesthetized. Two freshly harvested $\mathrm{R} 1$ tissue cubes of approximately $2 \mathrm{~mm}^{3}$ from the donor tumor were inserted into the apertures made in left and right lobes to induce bifocal liver tumors. Tumors reached a diameter of $0.8 \pm 0.2 \mathrm{~cm}$ after about 14 days were ready for use [19].

\section{Drug preparations}

CA4P (purchased from Toronto Research Chemicals, Canada, http://www.trc-canada.com/) was diluted in Phosphate buffered saline (PBS) solution at a concentration of $5 \mathrm{mg} / \mathrm{ml}$. Hyp purchased (PNP, Austrian. http://www.planta.at/hyper/ hyper.htm) has a purity $>98 \%$. Sodium iodides (123I and $\left.{ }^{131} \mathrm{I}\right)$ (supplied by MDS Nordion Inc., Fleurus, Belgium) have the radionuclide purities over $99 \%$.

Iodogen coating method was used for the radiolabeling. Briefly, a water-iosoluble oxidant "Iodogen" (1,3,4,6-tetrachloro-3a, 6a-diphenylglycouril) as iodination reagent is dissolved in an organic solvent and is coated on the walls of the glass reaction tube. 
Radioiodination is then initiated by the addition of dimethylsulfoxide (DMSO) solutions of Hyp and the neutralized solution of sodium Iodide hydroxide and, the mixture is incubated for 20 minute, and then terminated by removal of the reaction mixture. Determined by thin layer chromatography, the labeling efficiency above $99.5 \%$ was achieved with both ${ }^{123} \mathrm{I}-\mathrm{Hyp}$ and ${ }^{131} \mathrm{I}-\mathrm{Hyp}$. The ${ }^{131} \mathrm{I}-\mathrm{Hyp}$ has a specific activity typically $900 \mathrm{MBq} / \mathrm{mg}$ for therapy. Both tracers were re-dissolved in DMSO before injection.

\section{Experimental protocol}

As illustrated (Fig. 1), $10 \mathrm{mg} / \mathrm{kg}$ of CA4P was iv injected to all $\mathrm{R} 1$ models $24 \mathrm{~h}$ prior to radioiodinated Hyp. Three groups of 6 rats each containing 3 RLI and 3 R1 models received ${ }^{123}$ I-Hyp intravenously at 37, 74, and $185 \mathrm{MBq} / \mathrm{kg}$ respectively, and followed by $0.1 \mathrm{ml}$ of $1 \%$ Evans blue solution were sacrificed by groups at 4, 24 and $48 \mathrm{~h}$ post injection immediately after in vivo examination of magnetic resonance imaging (MRI) and planar scintigraphy/CT. Besides, two groups of 6 R1 models that received either ${ }^{131}$ I-Hyp at 300 $\mathrm{MBq} / \mathrm{kg}$ or vehicle $24 \mathrm{~h}$ after inducing tumor necrosis with CA4P were examined using MRI for comparisons of tumor growth for 12 days. Autoradiography, histopathology and/or gamma counting, were performed for postmortem verifications.

\section{In vivo imaging examinations}

MRI with T1-, T2-, and contrast-enhanced (CE)-T1 sequences were performed to determine the location and area of necrosis. Under isoflurane gas anesthesia, the rat placed in a wrist coil was scanned using a $1.5 \mathrm{~T}$ whole body MRI scanner (Sonata, Siemens, Erlangen, Germany) with iv bolus injection of Dotarem (Gd-DOTA, Guerbet, France) at 0.2 $\mathrm{mmol} / \mathrm{kg}$ for contrast enhancement [20]. After MRI, in vivo planar scintigraphy was performed for $2 \times 30$ minutes using a dual-head gamma camera (Biad XLT 25, Trionix Research Laboratories, Twinsburg, $\mathrm{OH}$, USA) equipped with high-energy collimators. The planar scintigraphy was immediately followed by a whole-body Computer tomography (CT) (Biograph16, Siemens, Knoxville, TN, USA) scan for co-registration. The final scintigraphic images were produced by summing the images of the two detector heads. A maximum intensity projection of the CT image was computed, providing the anatomical reference for the scintigraphic image. Both images were aligned with rigid registration based on mutual information.

\section{Regional biodistribution}

After being euthanized by overdose of pentobarbital, necrotic liver and tumor as well as normal liver were dissected and weighed. The necrotic liver and tumor tissues that were labeled by Evans blue were carefully sampled for gamma counting. Radioactivity was counted using a 3-inch NaI(T1) scintillation detector (Wallac Wizard,Turku, Finland). Tissues activities from RLI and R1 models were averaged by groups. Corrections were made for background radiation and physical decay during counting. Results were expressed as percentage of injected dose per gram of tissue (\%ID/g).

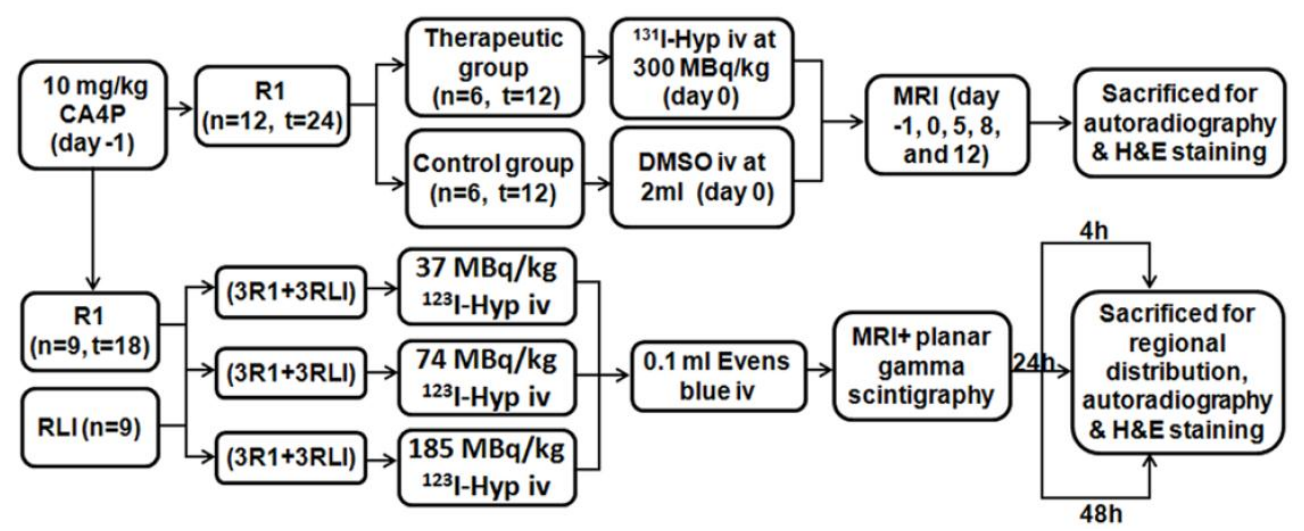

Figure I. Flowchart of experimental procedure. (n, number; $t$, tumor; RLI, rat models of reperfused liver infarction; RI, Rat models of hepatic allograft rhabdomyosarcoma). 


\section{Autoradiography}

After gamma counting, tissues were quickly frozen in isopentane-liquid nitrogen and cut with a cryotome (Microm HM 550, Walldorf, Germany) into 10-50 $\mu \mathrm{m}$ sections, and were thaw-mounted on glass slides. Frozen heads were digitally photographed after slicing. Autoradiograms of these slides were obtained by 24-48 hour exposure using a high-performance phosphor screen (super resolution screen; Canberra-Packard, Meriden, CT, USA) and the screens were read using a cyclone scanner with Optiquent software (Perkin Elmer, Waltham, Ma, USA). Relative tracer concentration in the necrotic tissues was estimated by regions of interest analysis and the results were compared with that from the viable tumor and liver.

\section{Histopathology and microscopes}

Slides were stained with Hematoxylin and Eosin (H\&E), examined using an optical microscope (Axioskop; Zeiss, Oberkochen, Germany) and digitally photographed.

\section{MR imaging analysis of tumoricidal effects}

Rats for comparisons of tumoricidal effects were scanned with MRI before and through 12 days after ${ }^{131}$ I-Hyp or vehicle injection. Tumor areas were manually delineated on each slice of T2-weighted sequence. Tumor volume was calculated according to the following formula: tumor volume $=\sum$ [tumor area on each slice $\times$ (slice thickness + gap)]. Tumor necrotic area was delineated from CE-T1 images as central non-enhancing region to generate tumor necrotic volume. Necrosis ratios were defined as the volume of necrosis over that of the entire tumor. Tumor doubling time (DT) was calculated use the equation: DT = $(\mathrm{T}-\mathrm{T} 0) \times \log 2 /(\log \mathrm{V}-\log \mathrm{V} 0)$, where $\mathrm{T}-\mathrm{T} 0$ indicates the time duration between the two measurements, and $\mathrm{V} 0$ and $\mathrm{V}$ denote the tumor volume at two points of measurements [20].

\section{Statistical analysis}

Numerical data was expressed as mean \pm standard deviation. Statistical analysis was performed using SPSS for Windows software package (version 16.0; SPSS, Chicago, IL, USA). An unpaired Student's t-test (two-tailed) was performed, $\mathrm{p}<0.05$ was considered statistically significant.

\section{Results}

\section{General aspects}

RLI and R1 tumor models were successfully established for this experiment. All rats survived the anesthesia, surgical procedures, tumor growth and in vivo imaging sessions. In all R1 models, CA4P induced intratumoral necrosis within a few hours after injection, but peripheral viable tumor tissue always co-existed as proven by MRI and histology.

\section{Quantification of necrosis avidity}

Gamma scintiscan showed initial systemic distribution of Hyp early after injection (within $4 \mathrm{~h}$ ). Considerable accumulations of radioactive in liver and tumor necrosis were evident with gamma counting from $4 \mathrm{~h}$ after injection. Radioactivity at 4, 24, and $48 \mathrm{~h}$ were $3.94 \pm 1.60,5.38 \pm 1.04$, and $6.03 \pm 2.09 \%$ $\mathrm{ID} / \mathrm{g}$ in necrotic liver, and $4.27 \pm 0.76,5.57 \pm 0.76$, and $5.68 \pm 1.33 \% \mathrm{ID} / \mathrm{g}$ in necrotic tumor, relative to $1.76 \pm$ $0.54,0.41 \pm 0.18$, and $0.16 \pm 0.07 \% \mathrm{ID} / \mathrm{g}$ in normal liver, with the mean necrosis-to-liver ratio around 2, 14 and 37 respectively (Table 1 ). Radioactivity measured from the necrotic liver and necrotic tumor showed no significant difference $(p>0.05)$ at the same time point. Radioactivity measured from the viable liver decreases continuously with time within $48 \mathrm{~h}$ $(p<0.01)$. Necrosis-to-liver ratios in both models increased continuously $(\mathrm{p}<0.001)$ within $48 \mathrm{~h}$.

Table I. Regional distribution of ${ }^{123}$ I-Hypericin $\left({ }^{123}\right.$ I-Hyp) in rats with reperfused liver infarction (RLI) and hepatic implanted rhabdomyosorcoma-I (RI). Necrosis-to-liver ratios derived from scintillation detector were corrected by the corresponding measurements from autoradiography. Data are expressed as mean of \% injected Dose/g $(\% / D / g) \pm S D$.

\begin{tabular}{llll}
\hline Organ/ ratio & \multicolumn{3}{c}{ \% injected dose per gram (\%ID/g) } \\
\cline { 2 - 4 } & $4 \mathrm{~h}$ & $24 \mathrm{~h}$ & $48 \mathrm{~h}$ \\
\hline $\mathrm{VL}$ & $1.76 \pm 0.54$ & $0.41 \pm 0.18 \downarrow$ & $0.16 \pm 0.07 \downarrow$ \\
$\mathrm{NL}^{*}$ & $3.94 \pm 1.60$ & $5.38 \pm 1.04$ & $6.03 \pm 2.09$ \\
$\mathrm{NT}^{*}$ & $4.27 \pm 0.76$ & $5.57 \pm 0.76$ & $5.68 \pm 1.33$ \\
$\mathrm{NL} /$ VL ratio & $2.23 \pm 0.44$ & $14.16 \pm 3.93 \uparrow$ & $37.58 \pm 2.40 \uparrow$ \\
$\mathrm{NT} /$ VL ratio & $2.55 \pm 0.75$ & $14.87 \pm 5.33 \uparrow$ & $36.45 \pm 6.44 \uparrow$ \\
\hline
\end{tabular}

VL: Viable Liver; NL: Necrotic Liver; NT: Necrotic Tumor. *: tissue that was stained by Evens Blue. $\downarrow$ : decrease significantly $(p<0.01)$ as compared with the previous measured time point. $\uparrow$ : increase significantly $(\mathrm{p}<0.001)$ as compared with the previous measured time point.

The necrotic liver lobe appeared homogeneously hypo- or iso-intense, but hyper-intense relative to the adjacent normal liver on T1 and T2 weighted MR images. On CE-T1 weighted image, reperfused necrotic liver lobe was hyper-enhanced by contrast agent (Fig. 2A).

On planar scintigrams, the radioactivity gradually accumulated in the liver and intestines, reflecting quick hepatic uptake and biliary excretion of ${ }^{123}$ I-Hyp. 
From $24 \mathrm{~h}$ after injection, hot spot of high uptake could be easily found in the liver region (Fig. 2B), revealing high accumulation of radioactivity in the necrotic lobe. The hot spot image corresponds closely to the hyper-enhanced liver lobe on CE-T1 MR images.

Macroscopically, Evans blue dye clearly marked the well-reperfusion necrotic tissue (Fig. $3 \mathrm{A1}^{\prime}-\mathrm{A3}^{\prime}$ ) in contrast to the unstained normal liver lobes (Fig. 3 A1A3) from the same animal, and imitated closely the autoradiography patterns of Hyp uptake (Fig. 3 B1'B3'). Normal liver lobes (Fig. 3 B1- B3) presented much less or negligible uptake of the radioactivity as compared with the necrotic part. The photographs of H\&E stained sections confirmed the presence of ne-

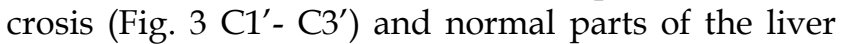
(Fig. 3 C1- C3), which were further proven by more powerful magnification views for the determination of viability (Fig. 3V) and necrosis (Fig. 3N). Cells that underwent necrotic process were characterized by the loss of glycogen particles, broken cell membranes, and discontinuous membranes, and appeared more eosinophilic due to an increase in cytosolic concentration of denatured proteins, which induce eosinophilia.

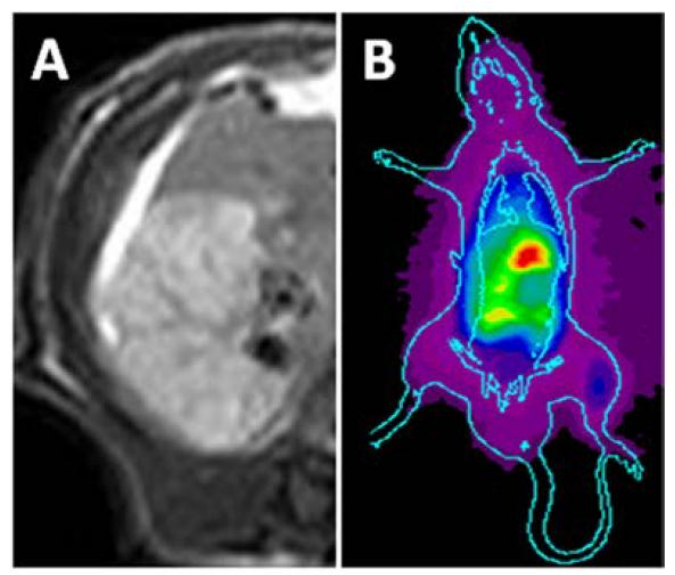

Figure 2. In vivo MRI and planar gamma imaging of necrosis in RLI models. Liver lobe with reperfused infarction showed homogeneous hyper-enhancement on CE-TI MR images (A). Planar gamma scintigraphy/radiography revealed a hotspot in the necrotic regions of the liver $(B)$.

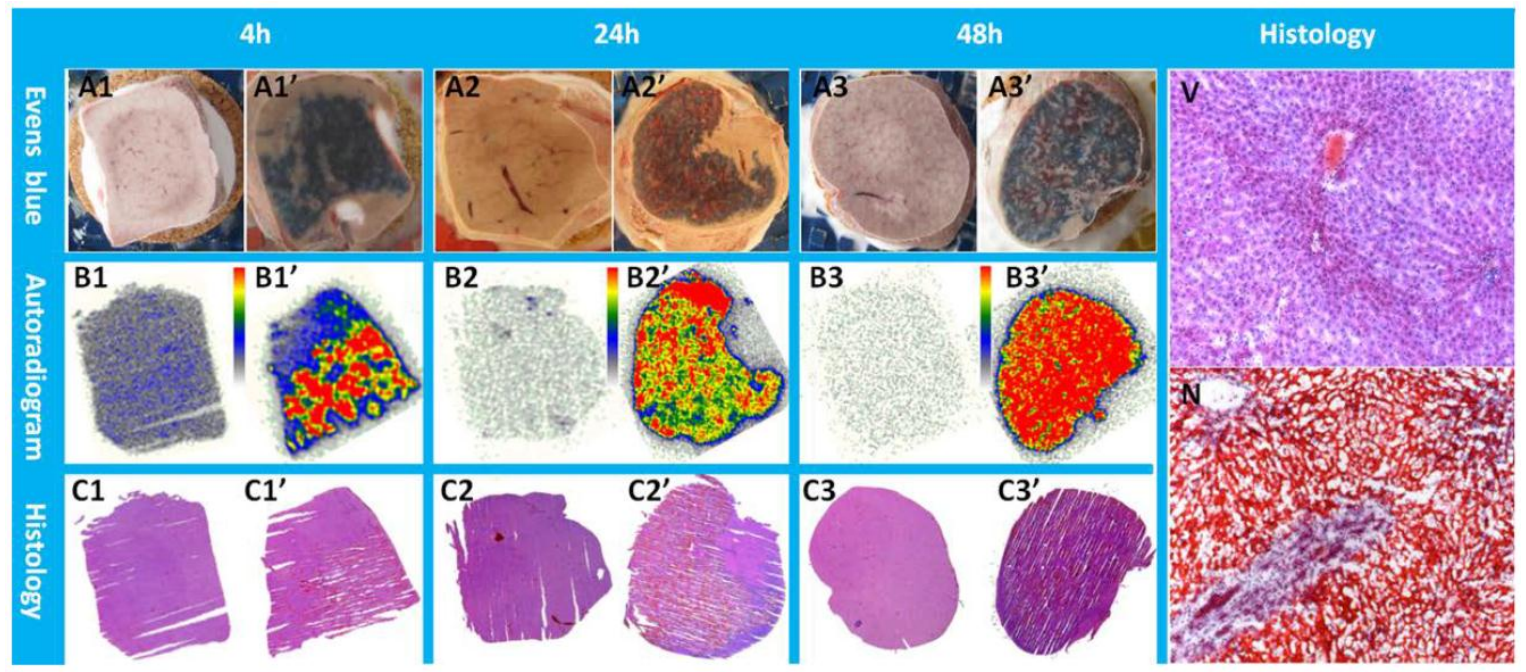

Figure 3. Typical pattern of Hyp uptake in necrotic liver in RLI models. Viable livers (A1, $A 2$, and $A 3)$ do not present blue appearance due to the quick washout of Evens blue dye. The presence of Evens blue clearly demonstrated the necrotic but well reperfused tissues (A1', A2', and A3'). From autoradiograms (B1, B2, B3, BI', B2', B3'), the necrotic liver (B1', B2', B3') revealed a much higher uptake of $1231-\mathrm{HyP}$ comparing with that of the viable liver (B1, B2, B3). The high radioactivity region seen on the autoradiogram presented a perfect match with the Evans blue stained areas, and has been further verified as necrosis on H\&E stained slices(C1, C2, C3, C1', C2', C3'). In viable liver sections $(\mathrm{V})$, hexagonal lobules can be found with well preserved architecture that consists of polygonal hepatocytes spreading out with their rounded nuclei as well as central veins. The sinusoids lined with endothelial cells were present in good order. In necrotic region (N) of the liver lobe, the border between hepatocytes became unclear due to membrane disintegration; the majority of the nuclei appeared atrophied and condensed. Destroyed erythrocytes were congested in and outside the sinusoidal space and central vein. Coagulation necrosis made the HE staining overall more eosinophilic. 


\section{Rat model of hepatic rhabdomyosarcoma al- lograft}

Similar to necrotic liver in RLI models, R1 tumors appeared homogeneously hyper-intense on T2 weighted MR images, but hypo-intense on plain T1 weighted images. On CE-T1 images, a hyper-enhanced rim embracing the unenhanced central necrosis was presented (Fig 4). Within hours after injection of CA4P, hemorrhagic necroses were induced in all R1 tumors as proven by CE-T1 MR imaging and histopathology (Fig. 5).

Although the diagnostic potential of radiolabeled Hyp derivatives for detecting tumors using single-photon emission computed tomography (SPECT) and positron emission tomography (PET) have been reported elsewhere $[8,13,21]$, liver R1 tumors could not be clearly contoured with planar scintigraphy within 48 hour post injection of ${ }^{123}$ I-Hyp in this study.
From tumor sections, a blue stained rim was found embracing the tumor which correlated well with the high radioactivity ring on the corresponding autoradiogram (Fig. 5). Necrosis-to-liver ratios defined by autoradiography were consistent with the outcomes from RLI models at the same time point, and in accordance with the quantitative results from gamma counting (Table 1). Hyp was absent deeper within necrotic zones on autoradiography due to the blocked access after complete vascular shutdown by CA4P. However, high amount of radioactivity was found centrally, occupying either partially or entirely the necrotic mass on day 12 (Fig. 4).

Photomicrographs of the H\&E stained sections showed that approximately 4-20 layers of viable tumor cells with the typical thickness about 20-100 $\mu \mathrm{m}$ in the periphery embracing the extensive central necrosis (Fig. 5). On 12 days post ${ }^{131} \mathrm{I}-\mathrm{Hyp}$, the viable rim became thicker (approximately $3-5 \mathrm{~mm}$ ) in tumors (Fig. 4).

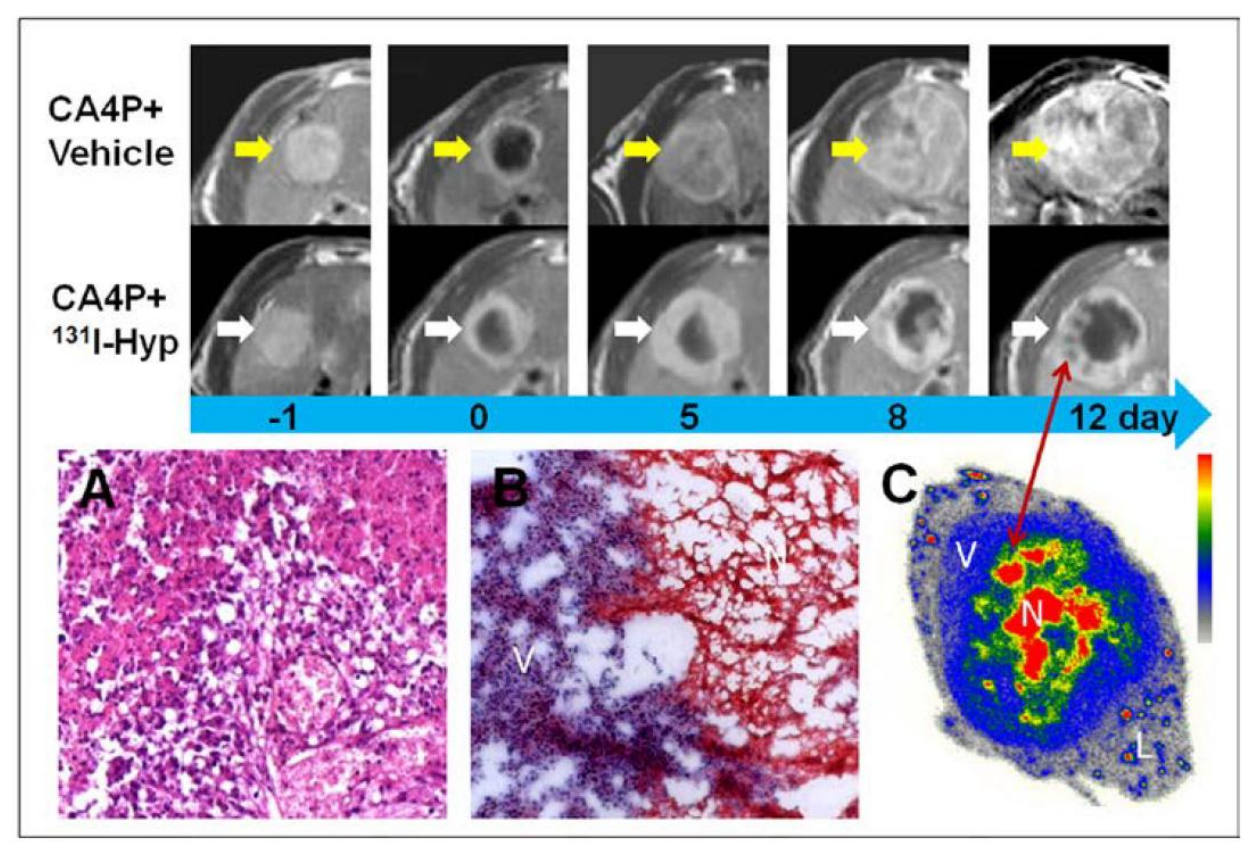

Figure 4. MR images of the representative tumors from the two groups showed the evolving tumor volume growth as well as the intratumoral necrosis. On day 0 , a hyper-intense rim can be seen surrounding the hypo-intense necrotic tumor. As compared with the CA4P control group, tumor growth in $131 \mathrm{I}-\mathrm{Hyp}$ was much slower with intratumoral necrosis remained or even outstretched till 12 days. Photomicrographs of $5 \mu \mathrm{m}$ tumor sections sampled from the interface between necrotic $(N)$ and viable tumor cells $(V)$ was shown $(A, B)$. In CA4P control group (A), the viable and dead cells, new vessels coexisted. lonizing irradiation of tumor cells exposed to $131 \mathrm{I}-\mathrm{H}$ yp caused marked cell death, characterized by cell membrane damage and extensive tumor damage (B). On I 2 days post $|3| \mid-H y p$, foci of radiation induced cell death was seen inside the viable tumor rim in MR images (red arrow), which was in correspondence with nests of relative high radioactivity in autoradiogram of the same section $(C)$. Liver $(L)$ showed much lower radioactivity as compared with the viable tumor $(V)$ and the intratumoral necrosis $(\mathrm{N})$ 


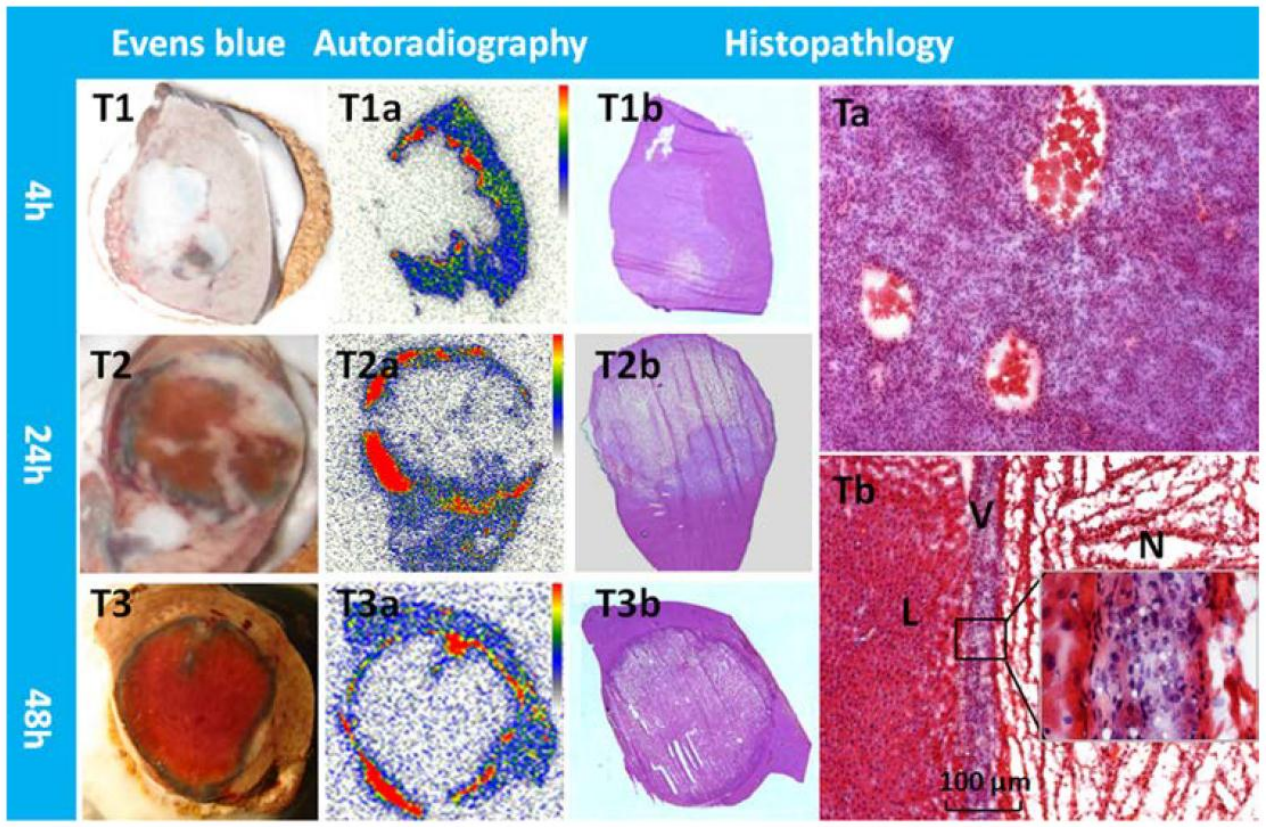

Figure 5. Typical patterns of Hyp uptake in necrotic tumor in RI models. Evens blue outlined the outer margin of tumor necrosis as a blue rim with viable tumor and normal liver much less stained $(\mathrm{T} 1, \mathrm{~T} 2, \mathrm{~T} 3)$. At all time points, a perfect match was found between the high radioactive ring on the autoradiogram $(\mathrm{T} 1 \mathrm{a}, \mathrm{T} 2 \mathrm{a}, \mathrm{T} 3 \mathrm{a})$ and the blue rim on Evens blue stained sections of the same tumor. H\&E stained macroscopy (T1b,T2b,T3b) and corresponding microscopy $(\mathrm{Ta}, \mathrm{Tb})$ confirmed the liver $(\mathrm{L})$ viable tumor $(\mathrm{V})$ and massive necrosis $(\mathrm{N})$. Following CA4P treatment, a few layers of viable tumor cells always existed in the periphery with the typical thickness less than $100 \mu \mathrm{m}$ (Tb).

\section{Tumoricidal effects in $\mathbf{R} \mathbf{I}$ models}

As shown in CE-T1 MR images (Fig. 4), despite the presence of initial vascular shutdown and necrosis, tumor grew rapidly in group of rats that received vehicle. Whereas in ${ }^{131} \mathrm{I}-\mathrm{Hyp}$ treated rats, tumor growth was much slower, with obvious unenhanced tumor center till 12 days. Tumor volumes in ${ }^{131} \mathrm{I}-\mathrm{Hyp}$ treated and vehicle control rats at $24 \mathrm{~h}$ post CA4P were $0.45 \pm 0.09 \mathrm{~cm}^{3}$, and $0.47 \pm 0.12 \mathrm{~cm}^{3}$ respectively ( $p>0.05)$; and $1.32 \pm 0.76$ and $3.63 \pm 0.72 \mathrm{~cm}^{3}(\mathrm{p}<$ 0.001 ) on day 12 , with the tumor DT $7.3 \pm 1.0$ and $4.2 \pm$ 0.7 days respectively. Tumor volume in vehicle control group almost tripled that of ${ }^{131} \mathrm{I}-\mathrm{Hyp}$ treated group over 12 days.

No intratumoral necrosis was evident before CA4P injection, whereas necrosis ratios as measured on MRI $24 \mathrm{~h}$ post CA4P were $73 \pm 12 \%$, and $76 \pm 17 \%$ in ${ }^{131} \mathrm{I}-\mathrm{Hyp}$ and vehicle control group ( $\left.p>0.05\right)$. On day 5 , the corresponding necrosis ratios were $61 \pm 17$ $\%$, and $27 \pm 14 \%$ with a significant difference between the two groups $(\mathrm{p}<0.001)$, and on day 12 after treatment, the necrosis ratios became $47 \pm 18 \%$ and $17 \pm 13$ $\%(\mathrm{p}<0.01)$ respectively. MR images and autoradiography indicated tumoricidal effects in ${ }^{131} \mathrm{I}-\mathrm{Hyp}$ treated tumors (Fig. 4). The evolving tumor volume growth and the corresponding necrosis ratios on day 0, 5, 8, 12 were also presented (Fig. 6).

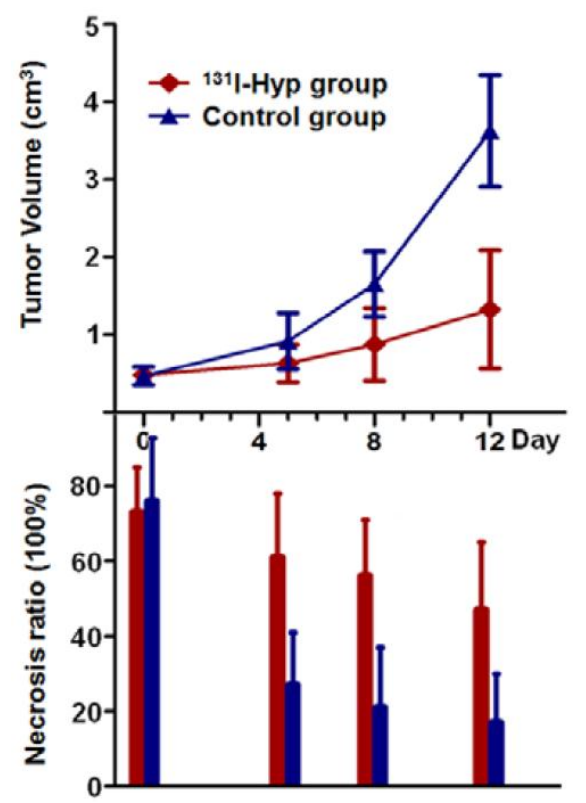

Figure 6. Tumor growth curve and the corresponding necrosis ratios on day $0,5,8,12$ post treatment. No significant difference was found in either tumor volume or tumor necrosis ratio between the two groups on day 0 . Tumor volume in $131 \mid-$ Hyp group was found significantly smaller than that of CA4P control group $(P$ $<0.01$ ) from day 5 on. Necrosis ratio measured from MR images presented a significant difference $(p<0.05)$ between the two groups from day 5 on. 


\section{Discussion}

RLI model was proven useful to study necrosis avid compounds; rodent model of R1 hepatic allograft provided a highly reproducible experimental tumor model with biologic features similar to those of human visceral hyper-vascular malignancies. Both RLI and R1 models can be noninvasively characterized morphologically by using a clinical MR imager and further validated postmortemly with golden standards. Iv injection of CA4P created a reliable means to induce massive necrosis noninvasively in solid tumor models [19,20,22].

Hyp belongs to a new category of target-specific agents and is deemed as an excellent option to develop different diagnostics for detecting necrosis-involved disorders, and radio-therapeutics for targeting solid malignancies [4,8-17]. Iodine-131 that processes both beta and gamma particle emissions may cause cell damage and is not suitable for pure diagnostic purpose in certain diseases, e.g., acute myocardial infarction and stroke. Hence both ${ }^{123}$ I-Hyp and ${ }^{131} \mathrm{I}-\mathrm{Hyp}$ have been evaluated with regard to their potential usefulness as necrosis targeting diagnostic or radio-therapeutic tracers. The conjugation of radioiodinated Hyp is relatively easy, more efficient and reproducible through an Iodogen coating method as compared with previously reported electrophilic substitution reaction [9].

Although the exact mechanisms behind necrosis avidity of Evans blue and Hyp are still to be elucidated, they were found to share similar affinity to necrotic tissues, though probably via different mechanisms of actions. It was hypothesized to be attributed to the binding of Hyp to phospholipids that are exposed to the exterior environment when the cell membrane is damaged [13], and the binding of Evans blue to albumin that deposits in the necrotic debris [23]. Systemic injection of Evans blue in animal models of pathologies provided a clear-cut distinction between viable and necrotic tissues, which is crucial for tissue stratification for biodistribution during macroscopic inspection (Fig. 3, Fig. 5).

For in vivo necrosis imaging using ${ }^{123} \mathrm{I}-\mathrm{Hyp}$, hot spots on planar gamma scintigraphy in RLI models were consistent with what were found in vivo by CE-T1 MRI (Fig. 2), and ex vivo by autoradiography, as well as Evens blue and H\&E staining (Fig. 3). With 123I-Hyp, the insufficient tumoral image within $48 \mathrm{~h}$ can be attributed to the poor microgeographical distribution or the no-entry of the radiotracer (Fig. 5), the considerable systemic retention of radioactivity at early stage, the rapid physical decay of iodine-123, as well as the relatively limited spatial resolution of planar gamma imaging, etc.. Nevertheless, in view of the vessel blocking effect caused by VDA, spontaneous or therapeutic necrosis other than VDA induced necrosis, e.g., chemotherapy, surgery, and other targeted cancer therapies, may present better blood perfusion for imaging of necrosis. Alternatively, for diagnostic purpose, long-lived radiotracer such as iodine-124 instead of iodine-123 in this setting would be advantageous, e.g., allows 4D imaging and quantification associated with microPET/CT scanning, and the animals could be scanned for up to 10 days post injection. Meanwhile, other imaging modalities such as SPECT or PET can be applied for improving the detection and quantification of radioactivity. Much more distinct hot spots only in tumoral regions have recently been acquired on scintigraphic images in mice with subcutaneous tumors 12 days after receiving ${ }^{131}$ I-Hyp intravenously (data to be published).

Systemic biodistributions of radioiodinated Hyp have been reported previously $[15,21,22,24,25]$. In this study we put emphasis on regional distributions of Hyp inside necrotic tissues as compared with the normal liver. Quantitative gamma counting revealed a progressive decrease of Hyp from elsewhere such as normal liver, but a continuous increase in necrotic-to-liver ratio over $48 \mathrm{~h}$. The high radioactive region with 123I-Hyp on autoradiogram matched well with the Evans blue stained region in both models, indicating the presence of necrosis as further proven by histopathologic findings. Necrotic-to-liver ratios derived from autoradiogram were somewhat even higher than that from gamma counting due to the impurity of necrotic tissues that were sampled for counting.

A few layers of remaining viable cells in the tumor rim persisted to repopulate and eventually led to tumor relapse after CA4P [26]. Therefore, special attention has been paid with SMSDTTS to combine VDA treatment with 131I-Hyp that targets and irradiates the tumor periphery. In this study we followed a longer period of 12 days (approximately 1.5 half-life of iodine-131). As shown on autoradiogram, the high activity rim was always closely surrounded by the peripheral viable rim, which provides an ideal solution for eliminating those remaining cells. The soft-tissue penetration range of iodine- 131 is $0.6-2.0$ $\mathrm{mm}$ [27], whereas the viable rim showed a typical thickness of less than $100 \mu \mathrm{m}$ (Fig. 5), which were within the irradiation range of the beta particles. High accumulation of ${ }^{131}$ I-Hyp allows the radiation dose in necrosis up to 100 times the cumulative dose needed for radiotherpeutic response [15]. As indicated by red arrow (Fig. 4), ${ }^{131}$ I-Hyp induced cell death may resemble the mulberry leaves that have been nibbled by 
silkworms, i.e. the multiple foci of radiation induced cell death emerged while the viable rim became increasingly thickened.

Although effective, tumor eradication was not achieved by the current single dose treatment in R1 models. This might be due to the following reasons: 1 ) the remaining tumor cells rapidly repopulate after VDA therapy [28]; 2) radiation normally induces apoptosis as another cell death type, which is slower and less radical comparing to necrosis [29]; 3) there is a lack of radioactivity that can continuously localize into the outstretched new necrotic tissues induced by the single dose of $\left.{ }^{131} \mathrm{I}-\mathrm{Hyp} ; 4\right)$ the newly developed tumor cells in the outer layer of the viable rim were less affected by the radiation; and 5) physical decay of radioiodine-131 inside necrosis. Therefore, consecutive doses might be necessary in future studies to further improve the anticancer efficacy with SMSDTTS. Meanwhile, other therapeutic radionuclides with longer half-life and/or higher-energy, i.e., alpha emitting isotopes; and deeper tissue penetration range, are supposed to be solutions to replace iodine-131 for improving cancer curability. In addition, anti-angiogenesis agents, which can prevent the formation of new blood vessels that feed the tumor, are supposed to provide synergistic effects for preventing tumor regrowth $[14,16]$.

This study confirmed the potential usefulness of radioiodinated Hyp for the assessment of tissue viability, detection of necrotic lesions, and the target-ability to malignancies as well. Hyp appears inherently superior to other existing agents in terms of its peculiar affinity for necrotic and irreversibly-damaged ischemic tissues [12]. The advantages of radioiodinated Hyp for clinical applications include: reliable effect, practical handling, affordable cost, suitable systemic administration, in vivo stability, and durable toxicity [30]. To further explore the theranostic potential of ${ }^{131} \mathrm{I}-\mathrm{Hyp}$ on malignancies, a more complete understanding of the mechanisms underlying necrosis avidity, the pharmacokinetics, and the in vivo stability are warranted. We anticipate that, upon optimization, the necrosis based theranostic tracer may prove a clinically useful drug in the near future.

\section{Conclusions}

Radioiodinated Hyp as a necrosis avid tracer appears promising for non-invasive imaging diagnosis of necrosis-related pathologies. Meanwhile, its prominent targetability to necrosis allows targeted radiotherapy for malignancies on top of a prior necrosis-inducing treatment.

\section{Abbreviation}

Hyp: Hypericin; RLI: Rat model of reperfused liver infarction; RI: Rat model of hepatic rhabdomyosarcoma; CA4P: Combretastatin A4 phosphate; NACAs: Necrosis avid contrast agents; SMSDTTS: Small-molecular sequential dual-targeting theragnostic strategy; VDA: Vascular disrupting agent; PBS: Phosphate buffered saline; DMSO: Dimethylsulfoxide; MRI: Magnetic resonance imaging; CE: Contrast-enhanced; CT: Computer tomography; H\&E: Hematoxylin and Eosin; DT: Doubling time; SPECT: Single-photon emission computed tomography; PET: positron emission tomography.

\section{Acknowledgments}

This work was partially supported by the grants awarded by FWO Vlaanderen ZWAP/05/018; Geconcerteerde Onderzoeksactie of the Flemish Government, OT project (OT/06/70); the KU Leuven Molecular Small Animal Imaging Center MoSAIC (KUL EF/05/08); the center of excellence In vivo Molecular Imaging Research (IMIR) of KU Leuven; the IWT SBO 'Imagine' (SBO80017), the National Natural Science Foundation of China (81071828), Jiangsu Province Natural Science Foundation (BK2010594) and a EU project Asia-Link CfP 2006-EuropeAid/ 123738/C/ACT/Multi-Proposal No. 128-498/111. The corresponding author Yicheng Ni is currently a Bayer Lecture Chair holder.

\section{Competing Interests}

We declare that we do not have any conflict of interest regarding the content of the paper. We have no financial relationship with any organizations that sponsored our research. We agree to allow the journal to review the data if requested.

\section{References}

1. Van Cruchten S, Van Den Broeck W. Morphological and biochemical aspects of apoptosis, oncosis and necrosis. Anat Histol Embryol. 2002;31:214-23.

2. Dasari M, Lee S, Sy J, et al. Hoechst-IR: an imaging agent that detects necrotic tissue in vivo by binding extracellular DNA. Org Lett. 2010;12:3300-3.

3. Walker NI, Harmon BV, Gobe GC, et al. Patterns of cell death. Methods Achiev Exp Pathol. 1988;13:18-54.

4. Ni Y, Bormans G, Chen F, et al. Necrosis avid contrast agents: functional similarity versus structural diversity. Invest Radiol 2005;40:526-35.

5. Flotats A, Carrio I. Non-invasive in vivo imaging of myocardial apoptosis and necrosis. Eur J Nucl Med Mol Imaging 2003;30:615-30.

6. Epstein A, Chen F, Taylor C. A novel method for the detection of necrotic lesions in human cancers. Cancer Res 1988; 48:5842-8.

7. Chen F, Taylor C, Epstein A. Tumor necrosis treatment of Me-180 human cervical carcinoma model with ${ }^{131}$ I-labeled TNT-1 monoclonal antibody. Cancer Res 1989;49:4578-85.

8. Ni Y, Huyghe D, Verbeke $\mathrm{K}$, et al. First preclinical evaluation of mono-[123I]iodohypericin as a necrosis-avid tracer agent. Eur J Nucl Med Mol Imaging 2006;33: 595 - 601. 
9. Fonge H, Vunckx K, Wang H, et al. Noninvasive detection and quantification of acute myocardial infarction in rabbits using mono[123I]iodohypericin microSPECT. Eur Heart J. 2008;29:260-269.

10. Ni Y, Bormans G, Marchal G, et al. Necrosis avid tracer agent. European patent EP1651201B1; January 2, 2008; Priority July 25, 2003.

11. Van de Putte M, Wang H, Chen F, et al. Hypericin as a marker for determination of tissue viability after radiofrequency ablation in a murine liver tumor model. Oncol Rep. 2008;19:927-932.

12. Van de Putte M, Ni Y, De Witte PA. Exploration of the mechanism underlying the tumor necrosis avidity of hypericin. Oncol Rep. 2008;19:921-6.

13. Song, S., Xiong, C., Zhou, M., et al. Small-animal PET of tumor damage induced by photothermal ablation with 64Cu-bis-DOTA-hypericin. J Nucl Med. 2011;52:792-9.

14. Cona MM, Wang $\mathrm{H}, \mathrm{Li}$ J, et al. Continuing pursuit for ideal systemic anticancer radiotherapeutics. Invest New Drugs. 2011; DOI: $10.1007 /$ s10637-011-9758-6.

15. Li J, Sun Z, Zhang J, et al. A dual-targeting anticancer approach: soil and seed principle. Radiology. 2011;260:799-807.

16. Li J, Chen F, Cona MM, et al. A Review on Various Targeted Anticancer Therapies. Target Oncol. Mar 2012;7:69-85. DOI: 10.1007/s11523-012-0212-2.

17. Van de Putte M, Marysael T, Fonge H, et al. Radiolabeled iodohypericin as tumor necrosis avid tracer: diagnostic and therapeutic potential. Int. J. Cancer: 2012; 131(2):E129-37. DOI: 10.1002/ijc.26492.

18. Wu X, Wang H, Chen F, et al. Rat model of reperfused partial liver infarction: characterization with multiparametric magnetic resonance imaging, microangiography, and histomorphology. Acta Radiol. 2009;50:276-87.

19. Chen F, Sun X, De Keyzer F, et al. Liver tumor model with implanted rhabdomyosarcoma in rats: MR imaging, microangiography, and histopathologic analysis. Radiology 2006; 239:554-62.

20. Wang H, Sun X, Chen F, et al. Treatment of rodent liver tumor with combretastatin a4 phosphate: noninvasive therapeutic evaluation using multiparametric magnetic resonance imaging in correlation with microangiography and histology. Invest Radiol. 2009;44:44-53.

21. Fonge H, Van de Putte M, Huyghe D, et al. Evaluation of tumor affinity of mono-[(123)I]iodohypericin and mono-[(123)I]iodoprotohypericin in a mouse model with a RIF-1 tumor. Contrast Media Mol Imaging. 2007;2:113-9.

22. Ni $Y$, Wang H, Chen F, et al. Tumor models and specific contrast agents for small animal imaging in oncology. Methods. 2009;48:125-38.

23. Hamer PW, McGeachie JM, Davies MJ, Grounds MD. Evans blue dye as an in vivo marker of myofibre damage: optimising parameters for detecting initial myofibre membrane permeability, J. Anat. 2002; 200: 69-79.

24. Bormans G, Huyghe D, Christiaen A, et al. Preparation, analysis and biodistribution in mice of iodine-123 labelled derivatives of hypericin. J Labelled Comp Rad. 2004;47:191-198.

25. Van De Putte M, Roskams T, Bormans G, et al. The impact of aggregation on the biodistribution of hypericin. Int J Oncol. 2006;28:655-60.

26. Tozer GM, Kanthou C, et al. Tumour vascular disrupting agents: combating treatment resistance. Br J Radiol. 2008;81:S12-S2.

27. Kelvin S.H Loke, Ajit K Padhy, David C.E Ng, et al. Dosimetric Considerations in Radioimmunotherapy and Systemic Radionuclide Therapies: A Review. World J Nucl Med. 2011;10:122-38.

28. Welford AF, Biziato D, Coffelt SB, et al. TIE2-expressing macrophages limit the therapeutic efficacy of the vascular-disrupting agent combretastatin A4 phosphate in mice. J Clin Invest. 2011;121:1969-1973.

29. Hofer KG. Heat potentiation of radiation damage versus radiation potentiation of heat damage. Radiat Res. 1987;110:450-7.

30. Li J, Miranda CM, Feng Y, Chen F, Zhang G, Fu X, Himmelreich U, Oyen R, Verbruggen A, Ni Y. A Single-dose Toxicity Study of Non-radioactive Iodinated Hypericin for a Targeted Anticancer Therapy in Mice. Acta Pharmacologica Sinica. 2012; Doi: 10.1038/aps.2012.111.

\section{Author Biography}

Dr. Yicheng Ni is a full professor at KU Leuven, Belgium. He holds the Bayer Lecture Chair and heads the Theragnostic Laboratory focusing on the topics related to life-threatening disorders including cancer and cardiovascular diseases. With his expertise in biomedical imaging, interventional therapy and contrast agent research, he has established a series of platforms for preclinical evaluation of diagnostics/therapeutics, attempted virtual biopsy for noninvasive characterization of liver tumors using contrast enhanced MRI, discovered necrosis-avid contrast agents (NACAs), invented a dual targeting cancer theragnostic strategy, trained tens of postgraduate students including the first $\mathrm{PhD}$ worldwide on cancer radiofrequency ablation (RFA), filed a dozen of patent applications, published numerous SCI journal papers and book chapters, edited/reviewed for many academic journals, worked as a guest professor in a number of foreign universities and institutes, coordinated/participated in several EU/national projects, served as an evaluation expert for organizations such as the European Commission (FP7), and received many academic honors including the prestigious Herbert M Stauffer Award (1993) and Elliott Lasser Award (2009) in the medical imaging field as well as the Editor's Recognition Award of Radiology for 7 consecutive years. Innovative, translational and longstanding research has ever been a pursuit of his academic career. 